\title{
Intuitionistic Fuzzy Topology and Intuitionistic Fuzzy Preorder
}

\author{
Sang Min Yun and Seok Jong Lee \\ Department of Mathematics, Chungbuk National University, Cheongju, Korea
}

\section{]jfis}

\begin{abstract}
This paper is devoted to finding relationship between intuitionistic fuzzy preorders and intuitionistic fuzzy topologies. For any intuitionistic fuzzy preordered space, an intuitionistic fuzzy topology will be constructed. Conversely, for any intuitionistic fuzzy topological space, we obtain an intuitionistic fuzzy preorder on the set. Moreover, we will show that the family of all intuitionistic fuzzy preorders on an underlying set has a very close link to the family of all intuitionistic fuzzy topologies on the set satisfying some extra condition.
\end{abstract}

Keywords: Intuitionistic fuzzy topology, Intuitionistic fuzzy preorder

\section{Introduction}

The theory of rough sets was introduced by Pawlak [1]. It is an extension of set theory for the research of intelligent systems characterized by insufficient and incomplete information. The relations between rough sets and topological spaces have been studied in some papers [2-4]. It is proved that the pair of upper and lower approximation operators is a pair of closure and interior of a topological space under a crisp reflexive and transitive relation.

The concept of fuzzy rough sets was proposed by replacing crisp binary relations with fuzzy relations in [5]. Furthermore in [6], the axiomatic approach for fuzzy rough sets were provided. In [7], the authors presented a general framework for the research of fuzzy rough sets in which both constructive and axiomatic approaches are used.

In [8], the authors showed that there is one to one correspondence between the family of fuzzy preorders on a nonempty set and the family of fuzzy topologies on this set satisfying certain extra conditions, and hence they are essentially equivalent.

This paper is devoted to finding relationship between intuitionistic fuzzy preorders and intuitionistic fuzzy topologies. For any intuitionistic fuzzy preordered space, an intuitionistic fuzzy topology will be constructed. Conversely, for any intuitionistic fuzzy topological space, we obtain an intuitionistic fuzzy preorder on the set. Moreover, we will show that the family of all intuitionistic fuzzy preorders on an underlying set has a very close link to the family of all intuitionistic fuzzy topologies on the set satisfying some extra condition.

\section{Preliminaries}

Let $X$ be a nonempty set. An intuitionistic fuzzy set $A$ is an ordered pair

$$
A=\left(\mu_{A}, \nu_{A}\right)
$$


where the functions $\mu_{A}: X \rightarrow I$ and $\nu_{A}: X \rightarrow I$ denote the degree of membership and the degree of nonmembership, respectively and $\mu_{A}+\nu_{A} \leq 1$ (See [9]). Obviously every fuzzy set $\mu$ in $X$ is an intuitionistic fuzzy set of the form $(\mu, \tilde{1}-\mu)$.

Throughout this paper, $I \otimes I$ denotes the family of all intuitionistic fuzzy numbers $(a, b)$ such that $a, b \in[0,1]$ and $a+b \leq 1$, with the order relation defined by

$$
(a, b) \leq(c, d) \text { iff } a \leq c \text { and } b \geq d
$$

And $\operatorname{IF}(X)$ denotes the family of all intuitionistic fuzzy sets in $X$, and 'IF' stands for 'intuitionistic fuzzy.'

Any IF set $A=\left(\mu_{A}, \nu_{A}\right)$ on $X$ can be naturally written as a function $A: X \rightarrow I \otimes I$ defined by $A(x)=\left(\mu_{A}(x), \nu_{A}(x)\right)$ for any $x \in X$.

For any IF set $A=\left(\mu_{A}, \nu_{A}\right)$ of $X$, the value

$$
\pi_{A}(x)=1-\mu_{A}(x)-\nu_{A}(x)
$$

is called an indeterminancy degree (or hesitancy degree) of $x$ to $A$ (See [9]). Szmidt and Kacprzyk [10] call $\pi_{A}(x)$ an intuitionistic index of $x$ in $A$. Obviously

$$
0 \leq \pi_{A}(x) \leq 1, \quad \forall x \in X
$$

Note $\pi_{A}(x)=0$ iff $\nu_{A}(x)=1-\mu_{A}(x)$. Hence any fuzzy set $\mu_{A}$ can be regarded as an IF set $\left(\mu_{A}, \nu_{A}\right)$ with $\pi_{A}=0$.

Definition 2.1 ( [11]). An IF set $R$ on $X \times X$ is called an $I F$ relation on $X$. Moreover, $R$ is called

(i) reflexive if $R(x, x)=(1,0)$ for all $x \in X$,

(ii) symmetric if $R(x, y)=R(y, x)$ for all $x, y \in X$,

(iii) transitive if $R(x, y) \wedge R(y, z) \leq R(x, z)$ for all $x, y, z \in$ $X$.

A reflexive and transitive IF relation is called an IF preorder. A symmetric IF preorder is called an IF equivalence. An IF preorder on $X$ is called an IF partial order if for any $x, y \in X$, $R(x, y)=R(y, x)=(1,0)$ implies that $x=y$. In this case, $(X, R)$ is called an IF partially ordered space. An IF preorder $R$ is called an IF equality if $R$ is both an IF equivalence and an IF partial order.

Remark 2.2. $R^{-1}$ is called the inverse of $R$ if $R^{-1}(x, y)=$ $R(y, x)$ for any $x, y \in X$. If $R$ is an IF preorder, so is $R^{-1} . R^{C}$ is called the complement of $R$ if $R^{C}(x, y)=\left(\nu_{R(x, y)}, \mu_{R(x, y)}\right)$ where $R(x, y)=\left(\mu_{R(x, y)}, \nu_{R(x, y)}\right)$. It is obvious that $R^{-1} \neq$ $R^{C}$.

Definition 2.3 ( [12]). Let $R$ be an IF relation on $X$. Then the two functions $\bar{R}, \underline{R}: \operatorname{IF}(X) \rightarrow \operatorname{IF}(X)$, defined by

$$
\begin{aligned}
& \bar{R}(A)(x)=\bigvee_{y \in X}(R(x, y) \wedge A(y)), \\
& \underline{R}(A)(x)=\bigwedge_{y \in X}\left(R^{C}(x, y) \vee A(y)\right),
\end{aligned}
$$

are called the upper approximation operator and the lower approximation operator on $X$, respectively. Moreover, $(X, R)$ is called an IF approximation space.

For any IF number $(a, b) \in I \otimes I,(\tilde{a}, \tilde{b})$ is an IF set which has the membership value a constant " $a$ " and the nonmembership value a constant " $b$ " for all $x \in X$.

Proposition 2.4 ( $[12,13])$. Let $(X, R)$ be an IF approximation space. Let $A, B \in \operatorname{IF}(X),\left\{A_{j} \mid j \in J\right\} \subseteq \operatorname{IF}(X)$ and $(a, b) \in$ $I \otimes I$. Then we have

(1) $\underline{R}((\tilde{1}, \tilde{0}))=(\tilde{1}, \tilde{0})$, $\bar{R}((\tilde{0}, \tilde{1}))=(\tilde{0}, \tilde{1})$,

(2) $A \subseteq B \Rightarrow \underline{R}(A) \subseteq \underline{R}(B)$, $\bar{R}(A) \subseteq \bar{R}(B)$,

(3) $\underline{R}\left(A^{C}\right)=(\bar{R}(A))^{C}$, $\bar{R}\left(A^{C}\right)=(\underline{R}(A))^{C}$,

(4) $\underline{R}(A \cap B)=\underline{R}(A) \cap \underline{R}(B)$, $\bar{R}(A \cup B)=\bar{R}(A) \cup \bar{R}(B)$,

(5) $\underline{R}\left(\bigcap_{j \in J} A_{j}\right)=\bigcap_{j \in J}\left(\underline{R}\left(A_{j}\right)\right)$, $\bar{R}\left(\bigcup_{j \in J} A_{j}\right)=\bigcup_{j \in J}\left(\bar{R}\left(A_{j}\right)\right)$,

(6) $\underline{R}((\tilde{a}, \tilde{b}) \cup A)=(\tilde{a}, \tilde{b}) \cup \underline{R}(A)$, $\bar{R}((\tilde{a}, \tilde{b}) \cap A)=(\tilde{a}, \tilde{b}) \cap \bar{R}(A)$.

Theorem $2.5([12,13])$. Let $(X, R)$ be an IF approximation space. Then

(1) $R$ is reflexive

$$
\begin{aligned}
& \Leftrightarrow \forall A \in \operatorname{IF}(X), \underline{R}(A) \subseteq A \\
& \Leftrightarrow \forall A \in \operatorname{IF}(X), A \subseteq \bar{R}(A) .
\end{aligned}
$$

(2) $R$ is transitive

$$
\begin{aligned}
& \Leftrightarrow \forall A \in \operatorname{IF}(X), \underline{R}(A) \subseteq \underline{R}(\underline{R}(A)) \\
& \Leftrightarrow \forall A \in \operatorname{IF}(X), \bar{R}(\bar{R}(A)) \subseteq \bar{R}(A) .
\end{aligned}
$$


Theorem 2.6. Let $(X, R)$ be a reflexive IF approximation space. If $\underline{R}\left(A_{j}\right)=A_{j}$ for each $j \in J$, then $\underline{R}\left(\bigcup_{j \in J} A_{j}\right)=$ $\bigcup_{j \in J} A_{j}$.

Proof By the reflexivity of $R$ and Theorem 2.5, $\underline{R}\left(\bigcup_{j \in J} A_{j}\right) \subseteq$ $\bigcup_{j \in J} A_{j}$. By Proposition 2.4,

$$
\underline{R}\left(\bigcup_{j \in J} A_{j}\right) \supseteq \bigcup_{j \in J} \underline{R}\left(A_{j}\right)=\bigcup_{j \in J} A_{j} .
$$

Thus $\underline{R}\left(\bigcup_{j \in J} A_{j}\right)=\bigcup_{j \in J} A_{j}$.

Example 2.7. Let $X=\left\{x_{1}, x_{2}\right\}$. Let $R=\left\{\left\langle\left(x_{1}, x_{1}\right), 1,0\right\rangle\right.$, $\left.\left\langle\left(x_{1}, x_{2}\right), 0.2,0.5\right\rangle,\left\langle\left(x_{2}, x_{1}\right), 0.4,0.3\right\rangle,\left\langle\left(x_{2}, x_{2}\right), 1,0\right\rangle\right\}$. Then $(X, R)$ is an IF approximation space. Let $A=\left\{\left\langle x_{1}, 0.6,0.3\right\rangle\right.$, $\left.\left\langle x_{2}, 0.5,0.4\right\rangle\right\}$ be an IF set on $X$, then

$\mu_{\bar{R}(A)}\left(x_{1}\right)=$

$$
\bigvee_{y \in X}\left(\mu_{R}\left(x_{1}, y\right) \wedge \mu_{A}(y)\right)=(1 \wedge 0.6) \vee(0.2 \wedge 0.5)=0.6
$$

$\nu_{\bar{R}(A)}\left(x_{1}\right)=$

$$
\bigwedge_{y \in X}\left(\nu_{R}\left(x_{1}, y\right) \vee \nu_{A}(y)\right)=(0 \vee 0.2) \wedge(0.5 \vee 0.4)=0.2 .
$$

Similarly, we obtain

$$
\mu_{\bar{R}(A)}\left(x_{2}\right)=0.5, \nu_{\bar{R}(A)}\left(x_{2}\right)=0.3 .
$$

Hence,

$$
\bar{R}(A)=\left\{\left\langle x_{1}, 0.6,0.2\right\rangle,\left\langle x_{2}, 0.5,0.3\right\rangle\right\} .
$$

Similarly, we have

$$
\underline{R}(A)=\left\{\left\langle x_{1}, 0.5,0.3\right\rangle,\left\langle x_{2}, 0.5,0.4\right\rangle\right\} .
$$

Proposition 2.8 ( [12]). For an IF relation $R$ on $X$ and $A \in$ $\operatorname{IF}(X)$, the pair $\bar{R}$ and $\underline{R}$ are "dual", i.e.,

$$
\begin{aligned}
& \bar{R}(A)=\left(\underline{R}\left(A^{C}\right)\right)^{C}, \\
& \underline{R}(A)=\left(\bar{R}\left(A^{C}\right)\right)^{C},
\end{aligned}
$$

where $A^{C}$ is the complement of $A$.

Definition 2.9 ( $[13,14])$. An IF topology $\mathcal{T}$ on $X$ in the sense of Lowen [15] is a family of IF sets in $X$ that is closed under arbitrary suprema and finite infima, and contains all constant IF sets. The IF sets in $\mathcal{T}$ are called open, and their complements, closed.

Definition 2.10 ( [8]). A Kuratowski IF closure operator on $X$ is a function $k: \operatorname{IF}(X) \rightarrow \operatorname{IF}(X)$ satisfying for $(a, b) \in$ $I \otimes I, A, B \in \operatorname{IF}(X)$,

(i) $k((\tilde{a}, \tilde{b}))=(\tilde{a}, \tilde{b})$,

(ii) $A \leq k(A)$,

(iii) $k(A \vee B)=k(A) \vee k(B)$,

(iv) $k(k(A))=k(A)$.

A Kuratowski IF closure operator $k$ on $X$ is called saturated if for all $A_{j} \in \operatorname{IF}(X), j \in J$,

$$
k\left(\bigvee\left\{A_{j} \mid j \in J\right\}\right)=\bigvee\left\{k\left(A_{j}\right) \mid j \in J\right\} .
$$

Furthermore, an IF topology is called saturated if it has a saturated IF closure operator.

Remark 2.11 ( [13]). Every Kuratowski IF closure operator $k$ on $X$ gives rise to an IF topology on $X$ in which an IF set $B$ is closed iff $k(B)=B$.

\section{Intuitionistic Fuzzy Implication Operator}

Generally, for $\left(a_{1}, a_{2}\right),\left(b_{1}, b_{2}\right) \in I \otimes I$, the implication operator (or residual implicator) $[16,17]$ is defined as follows;

$$
\begin{aligned}
& \left(a_{1}, a_{2}\right) \rightarrow\left(b_{1}, b_{2}\right) \\
& =\sup \left\{\left(d_{1}, d_{2}\right) \in I \otimes I \mid\left(a_{1}, a_{2}\right) \wedge\left(d_{1}, d_{2}\right) \leq\left(b_{1}, b_{2}\right)\right\} .
\end{aligned}
$$

If given IF numbers $\left(a_{1}, a_{2}\right),\left(b_{1}, b_{2}\right)$ are comparable, then the IF implication operator is clearly given by

$$
\left(a_{1}, a_{2}\right) \rightarrow\left(b_{1}, b_{2}\right)= \begin{cases}(1,0) & \text { if } \quad\left(a_{1}, a_{2}\right) \leq\left(b_{1}, b_{2}\right) \\ \left(b_{1}, b_{2}\right) & \text { if } \quad\left(a_{1}, a_{2}\right)>\left(b_{1}, b_{2}\right)\end{cases}
$$

for all $\left(a_{1}, a_{2}\right),\left(b_{1}, b_{2}\right) \in I \otimes I$.

But it is not always able to compare given IF numbers. Nevertheless, in many papers the IF implication operator is studied where given IF numbers are comparable. In this paper, we consider the IF implication operator to the extend that the given IF numbers are not comparable with some restrictions. 
Remark 3.1. The IF residual implicator is clearly given by $\left(a_{1}, a_{2}\right) \rightarrow\left(b_{1}, b_{2}\right)=\left\{\begin{array}{lll}(1,0) & \text { if }\left(a_{1}, a_{2}\right) \leq\left(b_{1}, b_{2}\right), \\ \left(b_{1}, b_{2}\right) & \text { if }\left(a_{1}, a_{2}\right)>\left(b_{1}, b_{2}\right), \\ \left(1-b_{2}, b_{2}\right) & \text { if } a_{1}<b_{1}, a_{2} \leq b_{2}, \\ \left(b_{1}, 1-b_{1}\right) & \text { if } a_{1}>b_{1}, a_{2} \geq b_{2},\end{array}\right.$ for all $\left(a_{1}, a_{2}\right),\left(b_{1}, b_{2}\right) \in I \otimes I$.

Definition 3.2. (1) For $\left(a_{1}, a_{2}\right) \in I \otimes I, A \in \operatorname{IF}(X)$ and $x \in$ $X$, the map $\left(\left(a_{1}, a_{2}\right) \wedge A\right): I \otimes I \times \operatorname{IF}(X) \rightarrow \operatorname{IF}(X)$ is defined by

$$
\left(\left(a_{1}, a_{2}\right) \wedge A\right)(x)=\left(a_{1}, a_{2}\right) \wedge A(x) .
$$

(2) For $\left(a_{1}, a_{2}\right) \in I \otimes I, A \in \operatorname{IF}(X)$ and $x \in X$, the map $\left(\left(a_{1}, a_{2}\right) \rightarrow A\right): I \otimes I \times \operatorname{IF}(X) \rightarrow \operatorname{IF}(X)$ is defined by

$$
\left(\left(a_{1}, a_{2}\right) \rightarrow A\right)(x)=\left(a_{1}, a_{2}\right) \rightarrow A(x) .
$$

(3) For $A, B \in \operatorname{IF}(X)$, the map $(A \rightarrow B): \operatorname{IF}(X) \times \operatorname{IF}(X) \rightarrow$ $\operatorname{IF}(X)$ is defined by

$$
(A \rightarrow B)(x)=A(x) \rightarrow B(x), \quad \forall x \in X .
$$

Remark 3.3. (1) We denote by $\left[\left(a_{1}, a_{2}\right),(1,0)\right]$ the rectangular plane which represents $\left[a_{1}, 1\right] \times\left[0, a_{2}\right]$. For a set $A \subseteq X$, an IF set $\chi_{A}: X \rightarrow I \otimes I$ is a map defined by

$$
\chi_{A}(x)= \begin{cases}(1,0) & \text { if } x \in A, \\ (0,1) & \text { if } x \notin A .\end{cases}
$$

From the above, if $\left(a_{1}, a_{2}\right) \in I \otimes I$ and $A \in \operatorname{IF}(X)$ are comparable, then we have that

$$
\left(a_{1}, a_{2}\right) \rightarrow A=\chi_{A^{-1}\left[\left(a_{1}, a_{2}\right),(1,0)\right]} \vee A .
$$

(2) If $\left(a_{1}, a_{2}\right),\left(b_{1}, b_{2}\right),\left(c_{1}, c_{2}\right) \in I \otimes I$ are all comparable, then

$$
\begin{gathered}
\left(a_{1}, a_{2}\right) \wedge\left(b_{1}, b_{2}\right) \leq\left(c_{1}, c_{2}\right) \\
\operatorname{iff}\left(b_{1}, b_{2}\right) \leq\left(a_{1}, a_{2}\right) \rightarrow\left(c_{1}, c_{2}\right) .
\end{gathered}
$$

Clearly the following holds;

$$
\begin{aligned}
\left(\left(a_{1}, a_{2}\right)\right. & \left.\rightarrow\left(b_{1}, b_{2}\right)\right) \wedge\left(\left(b_{1}, b_{2}\right) \rightarrow\left(c_{1}, c_{2}\right)\right) \\
& \leq\left(\left(a_{1}, a_{2}\right) \rightarrow\left(c_{1}, c_{2}\right)\right) .
\end{aligned}
$$

Theorem 3.4. The implication operator " $\rightarrow$ " is an IF preorder on $I \otimes I$.

Proof By the above property and the fact

$$
\left(a_{1}, a_{2}\right) \rightarrow\left(a_{1}, a_{2}\right)=(1,0),
$$

it follows.

\section{Intuitionistic Fuzzy Preorder and Intuitionis- tic Fuzzy Topology}

Let $(X, \leq)$ be a preordered space and $A \subseteq X$. Let $\uparrow A=$ $\{y \in X \mid y \geq x$, for some $x \in A\}$. If $\uparrow A=A$, then $A$ is called an upper set. Dually if $B=\downarrow B=\{y \in X \mid$ $y \leq x$, for some $x \in B\}$, then $B$ is called a lower set. The family of all the upper sets of $X$ is clearly a topology on $X$, which is called the Alexandrov topology (See [18]) on $X$, and denoted $\Gamma(\leq)$. We write simply $\Gamma(X)$ for the topological space $(X, \Gamma(\leq))$.

On the other hand, for a topological space $(X, \mathcal{T})$ and $x, y \in$ $X$, let $x \leq y$ if $x \in U$ implies $y \in U$ for any open set $U$ of $X$, or equivalently, $x \in \overline{\{y\}}$. Then $\leq$ is a preorder on $X$, called the specialization order (See [18]) on $X$. Denote this preorder by $\Omega(\mathcal{T})$. We also write simply $\Omega(X)$ for $(X, \Omega(\mathcal{T}))$.

A function $f:\left(X, \leq_{1}\right) \rightarrow\left(Y, \leq_{2}\right)$ between two preordered sets is called order-preserving if $x \leq_{1} y$ implies $f(x) \leq_{2} f(y)$.

From now on we are going to enlarge the above ideas to the IF theories in a natural way.

Definition 4.1. Let $(X, R)$ be an IF preordered space. Then $A \in \operatorname{IF}(X)$ is called an IF upper set in $(X, R)$ if

$$
A(x) \wedge R(x, y) \leq A(y), \quad \forall x, y \in X .
$$

Dually, $A$ is called an $I F$ lower set if $A(y) \wedge R(x, y) \leq A(x)$ for all $x, y \in X$.

Let $R$ be an IF preorder on $X$. For $x, y \in X$, the real number $R(x, y)$ can be interpreted as the degree to which $x$ is less than or equal to $y$. The condition $A(x) \wedge R(x, y) \leq A(y)$ can be interpreted as the statement that if $x$ is in $A$ and $x \leq y$ then $y$ is in $A$. Particularly, if $R$ is an IF equivalence relation, then an IF set $A$ is an upper set in $(X, R)$ if and only if it is a lower set in $(X, R)$.

The classical preorder relation $x \leq y$ can be naturally extended to $R(x, y)=(1,0)$ in IF preorder relation. Since 
$(1,0)=R(x, y) \leq A(x) \rightarrow A(y), A(x) \leq A(y)$ for any IF upper set $A$. That is, $x \leq y$ means $A(x) \leq A(y)$. Obviously, the notion of IF upper sets and IF lower sets agrees with that of upper sets and lower sets in classical preordered space.

Definition 4.2. A function $f:\left(X, R_{1}\right) \rightarrow\left(Y, R_{2}\right)$ between IF preordered spaces is called order-preserving if

$$
R_{1}(x, y) \leq R_{2}(f(x), f(y)), \forall x, y \in X
$$

Definition 4.3. Let $X$ be a totally ordered set. An IF set $A$ on $X$ is called

(i) increasing if for all $x, y \in X$ with $x<y, A(x) \leq A(y)$,

(ii) decreasing if for all $x, y \in X$ with $x<y, A(x) \geq A(y)$,

(iii) monotone if $A$ is increasing or decreasing.

Definition 4.4. Let $A=\left(\mu_{A}, \nu_{A}\right)$ be an IF set. $A$ is called simple if $\mu_{A}$ or $\nu_{A}$ is a constant function.

Remark 4.5. (1) If indeterminancy degree $\pi_{A}$ is a constant function $t$, then for any two different elements $x, y \in X$, $\pi_{A}(x)=\pi_{A}(y)=t$. So, if $\mu_{A}(x) \leq \mu_{A}(y)$, then $\nu_{A}(x) \geq$ $\nu_{A}(y)$. Hence $A(x) \leq A(y)$. Therefore $A(x)$ and $A(y)$ are comparable for any $x, y \in X$ provided that the hesitancy degree of an IF set $A$ is a constant function.

(2) Suppose that the universal set $X$ is a totally ordered set. If $A$ is monotone or simple, then $A(x)$ and $A(y)$ are comparable for any different element $x, y \in X$.

From now on, in order to avoid getting imprecise value in acting with the implication operator, we will consider only the IF set $A: X \rightarrow I \otimes I$ such that $A(x)$ and $A(y)$ are comparable for any $x, y \in X$. By Remark 4.5, any function which is monotone or simple or of constant hesitancy degree is an example of such function.

Lemma 4.6. For a given IF preordered space $(X, R)$, an IF set $B: X \rightarrow I \otimes I$ is an IF upper set of $(X, R)$ if and only if $B:(X, R) \rightarrow(I \otimes I, \rightarrow)$ is an order-preserving function.

Proof For any $B \in \operatorname{IF}(X)$, we have the following relations.

$B$ is an IF upper set in $(X, R)$

$$
\begin{array}{ll}
\Leftrightarrow & B(x) \wedge R(x, y) \leq B(y) \text { for all } x, y \in X \\
\Leftrightarrow & R(x, y) \leq B(x) \rightarrow B(y) \text { for all } x, y \in X \\
\Leftrightarrow & \text { The map } B:(X, R) \rightarrow(I \otimes I, \rightarrow) \text { defined by } \\
& x \rightsquigarrow B(x)=\left(\mu_{B}(x), \nu_{B}(x)\right) \text { preserves order. }
\end{array}
$$

Theorem 4.7. If $(X, R)$ is an IF preordered space, then the family $\mathcal{T}$ of all the upper sets in $X$ satisfies the following conditions, and hence it is an IF topology on $X$.

For any IF sets $A_{j}, A \in \mathcal{T}$;

(i) $\forall(a, b) \in I \otimes I,(\tilde{a}, \tilde{b}) \in \mathcal{T}$,

(ii) $\bigvee_{j \in J} A_{j} \in \mathcal{T}$,

(iii) $\bigwedge_{j \in J} A_{j} \in \mathcal{T}$,

(iv) $\forall(a, b) \in I \otimes I,((a, b) \wedge A) \in \mathcal{T}$,

(v) Suppose that for any $A \in \mathcal{T}$ and $x, y \in X$ and $(a, b) \in$ $I \otimes I, A(x)$ and $A(y)$ and $(a, b)$ are all comparable. Then $((a, b) \rightarrow A) \in \mathcal{T}$.

Proof (i) Let $(a, b) \in I \otimes I$, then $(\tilde{a}, \tilde{b})(x) \wedge R(x, y)=(a, b) \wedge$ $R(x, y) \leq(a, b)=(\tilde{a}, \tilde{b})(y)$. So $(\tilde{a}, \tilde{b}) \in \mathcal{T}$.

(ii) $\left(\bigvee A_{j}\right)(x) \wedge R(x, y)=\bigvee\left(A_{j}(x) \wedge R(x, y)\right) \leq \bigvee\left(A_{j}(y)\right)$ $=\left(\bigvee A_{j}\right)(y)$.

(iii) $\left(\bigwedge A_{j}\right)(x) \wedge R(x, y)=\bigwedge\left(A_{j}(x) \wedge R(x, y)\right) \leq \bigwedge\left(A_{j}(y)\right)$ $=\left(\bigwedge A_{j}\right)(y)$.

(iv) If $A$ is an upper set, then $A(x) \wedge R(x, y) \leq A(y)$ for any $x, y \in X$. So, we obtain that

$$
((a, b) \wedge A(x)) \wedge R(x, y) \leq((a, b) \wedge A(y)),
$$

for all $(a, b) \in I \otimes I$. This means that $((a, b) \wedge A) \in \mathcal{T}$.

(v) By Remark 3.3, $((a, b) \rightarrow A(x)) \wedge(A(x) \rightarrow A(y)) \leq$ $((a, b) \rightarrow A(y))$. Thus $((a, b) \rightarrow A(x)) \rightarrow((a, b) \rightarrow A(y)) \geq$ $(A(x) \rightarrow A(y)) \geq R(x, y)$. Hence $((a, b) \rightarrow A(x)) \wedge R(x, y)$ $\leq((a, b) \rightarrow A(y))$.

Definition 4.8. For an IF preordered space $(X, R)$, let

$$
\begin{aligned}
& \mathcal{T}_{\bar{R}}=\{A \in \operatorname{IF}(X) \mid A=\bar{R}(A)\}, \\
& \mathcal{T}_{\underline{R}}=\{A \in \operatorname{IF}(X) \mid A=\underline{R}(A)\} .
\end{aligned}
$$

If $\mathcal{T}$ is a family of IF sets in $X$ which satisfies conditions (i)-(v) of Theorem 4.7, then there exists an IF preorder $R$ on $X$ such that $\mathcal{T}$ consists of all the upper sets of the IF preordered space $(X, R)$. It will be shown in the following theorem.

Lemma 4.9. Let $\Lambda$ be a subfamily of $\operatorname{IF}(X)$ such that for any $A \in \Lambda$ and for any $x, y \in X, A(x)$ and $A(y)$ are comparable. Let $R(x, y)=\bigwedge_{B \in \Lambda}(B(x) \rightarrow B(y))$. Then $R$ is an IF preorder. 
Proof (i) $R(x, x)=\bigwedge_{B \in \Lambda}(B(x) \rightarrow B(x))=(1,0)$.

(ii) $R(x, y) \wedge R(y, z)=\left(\bigwedge_{B \in \Lambda}(B(x) \rightarrow B(y))\right) \wedge\left(\bigwedge_{D \in \Lambda}\right.$ $(D(y) \rightarrow D(z))) \leq \bigwedge_{B \in \Lambda}((B(x) \rightarrow B(y)) \wedge(B(y) \rightarrow$ $B(z))) \leq \bigwedge_{B \in \Lambda}(B(x) \rightarrow B(z))=R(x, z)$.

Theorem 4.10. Let $\Lambda$ be a subfamily of $\operatorname{IF}(X)$ satisfying (i)-(v) of Theorem 4.7 such that for any $A \in \Lambda$ and for any $x, y \in X$, $A(x)$ and $A(y)$ are comparable. Then there exists an IF preorder $R$ such that $\Lambda$ is the family $\mathcal{T}$ of all upper sets in $X$ with respect to $R$.

Proof Suppose that $\Lambda \subseteq \operatorname{IF}(X)$ satisfy the conditions (i)-(v) of Theorem 4.7. Define $R(x, y)=\bigwedge_{B \in \Lambda}(B(x) \rightarrow B(y))$. By the above lemma, $R$ is an IF preorder on $X$. Let $B \in \Lambda$. Since $B(x)$ and $B(y)$ are comparable for any $x, y \in X$, we have $R(x, y) \leq B(x) \rightarrow B(y)$. Hence $B(x) \wedge R(x, y) \leq B(y)$, i.e. $B$ is an IF upper set. Thus $\Lambda \subseteq \mathcal{T}$.

What remains is to show that $\mathcal{T} \subseteq \Lambda$. Take $D \in \mathcal{T}$. For a given $x \in X$, define $m_{x}: X \rightarrow I \otimes I$ by $m_{x}(z)=D(x) \wedge$ $R(x, z)$ for all $z \in X$. Then $m_{x}(x)=D(x)$ and $m_{x}(z) \leq$ $D(z)$ for all $z \in X$. Thus $D=\bigvee_{x \in X} m_{x}$.

For each $B \in \Lambda$ and previously given $x$, define $g_{B}: X \rightarrow$ $I \otimes I$ by $g_{B}(z)=B(x) \rightarrow B(z)$ for all $z \in X$. By (v), $g_{B} \in \Lambda$. By (iii), we obtain that $\bigwedge_{B \in \Lambda} g_{B} \in \Lambda$. Since

$$
m_{x}(z)=D(x) \wedge R(x, z)=D(x) \wedge\left(\bigwedge_{B \in \Lambda}(B(x) \rightarrow B(z))\right.
$$

$=D(x) \wedge\left(\bigwedge_{B \in \Lambda} g_{B}(z)\right)$,

we have $m_{x} \in \Lambda$. Note that $D=\bigvee_{x \in X} m_{x}$. Therefore $D \in \Lambda$ by (ii).

Example 4.11. Let $X=[0,1]$ be the universal set. Let $\Lambda$ be the family of all constant IF sets on $X$ and the IF set $A=\left(\mu_{A}, \nu_{A}\right)$, where $\mu_{A}(x)=1-x, \nu_{A}(x)=x$. Take $\Gamma$ by arbitrary suprema and arbitrary infima with members of $\Lambda$. Then clearly $\Gamma \subseteq$ $\operatorname{IF}(X)$ and it satisfies (i)-(v) of Theorem 4.7. We can define the order $R$ on $X$ by

$$
R(x, y)=\bigwedge_{B \in \mathcal{T}}(B(x) \rightarrow B(y)) \text { for all } x, y \in X .
$$

Then clearly $R$ is reflexive. Take $x, y \in X$ such that $x \leq y$, then $R(x, y)=A(y)$ and $R(y, x)=(1,0)$. So we know that $R$ is transitive. Therefore $R$ is a preorder on $X$. Consider $A(x) \wedge R(x, y)$ when $x \leq y$. Then $R(x, y)=A(y)$, and hence $A(x) \wedge R(x, y)=A(x) \wedge A(y) \leq A(y)$. Consequently we know that $\Gamma$ is the family of all upper sets in $X$ with respect to $R$.

The following result relates lower sets and upper sets in an IF preordered space $(X, R)$ with the upper approximation operator $\bar{R}$ and $\overline{R^{-1}}$, respectively.

Proposition 4.12. Let $(X, R)$ be an IF preordered space and $A \in \operatorname{IF}(X)$. Then $A$ is a lower set iff $A=\bar{R}(A)$.

Proof $A$ is a lower set

$$
\begin{aligned}
& \text { iff } A(y) \wedge R(x, y) \leq A(x), \forall x, y \in X \\
& \text { iff } \bigvee\{A(y) \wedge R(x, y) \mid y \in X\} \leq A(x), \forall x \in X \\
& \text { iff } \bar{R}(A)(x) \leq A(x), \forall x \in X \\
& \text { iff } \bar{R}(A) \leq A \\
& \text { iff } \bar{R}(A)=A .
\end{aligned}
$$

Proposition 4.13. Let $(X, R)$ be an IF preordered space and $A \in \operatorname{IF}(X)$. Then $A$ is an upper set iff $A=\overline{R^{-1}}(A)$.

Proof $A$ is an upper set

$$
\begin{aligned}
& \text { iff } A(x) \wedge R(x, y) \leq A(y), \forall x, y \in X \\
& \text { iff } \bigvee\{A(x) \wedge R(x, y) \mid x \in X\} \leq A(y), \forall y \in X \\
& \text { iff } \bigvee\left\{A(x) \wedge R^{-1}(y, x) \mid x \in X\right\} \leq A(y), \forall y \in X \\
& \text { iff } \overline{R^{-1}}(A)(y) \leq A(y), \forall y \in X \\
& \text { iff } \overline{R^{-1}}(A) \leq A \\
& \text { iff } \overline{R^{-1}}(A)=A .
\end{aligned}
$$

Remark 4.14. Let $\mathcal{T}$ be the family of all upper sets of an IF preordered space $(X, R)$. By Theorem 4.7, $\mathcal{T}$ is an Alexandrov IF topology on $X$. Furthermore $\mathcal{T}=\mathcal{T}_{\overline{R^{-1}}}$ by the above proposition.

Proposition 4.15. Let $(X, R)$ be an IF preordered space. Then $\mathcal{T}_{\underline{R}}=\{A \in \operatorname{IF}(X) \mid A=\underline{R}(A)\}$ is an Alexandrov IF topology on $X$.

Proof (1) Take $(a, b) \in I \otimes I$, then we know that $\underline{R}((\tilde{a}, \tilde{b}))(x)=$ $\bigwedge_{y \in X}\left((\tilde{a}, \tilde{b})(y) \vee R^{C}(x, y)\right) \geq \bigwedge_{y \in X}(\tilde{a}, \tilde{b})(y)=(a, b)=$ 
$(\tilde{a}, \tilde{b})(x)$. So $\underline{R}((\tilde{a}, \tilde{b})) \geq(\tilde{a}, \tilde{b})$. Clearly $\underline{R}((\tilde{a}, \tilde{b}) \leq(\tilde{a}, \tilde{b})$. Hence $(\tilde{a}, \tilde{b})=\underline{R}((\tilde{a}, \tilde{b}))$. Therefore $(\tilde{a}, \tilde{b}) \in \mathcal{T}_{\underline{R}}$ for any $(a, b) \in I \otimes I$.

(2) Take $A_{j} \in \mathcal{T}_{\underline{R}}$. Then $A_{j}=\underline{R}\left(A_{j}\right)$ for each $j \in J$. So $\left(\bigwedge_{j \in J} A_{j}\right)(x)=\left(\bigwedge_{j \in J} \underline{R}\left(A_{j}\right)\right)(x)=\bigwedge_{j \in J} \underline{R}\left(A_{j}\right)(x)=$ $\bigwedge_{j \in J}\left(\bigwedge_{y \in X}\left(A_{j}(y) \vee R^{C}(x, y)\right)\right)=\bigwedge_{y \in X}\left(\left(\bigwedge_{j \in J} A_{j}\right)(y) \vee\right.$ $\left.R^{C}(x, y)\right)=\underline{R}\left(\bigwedge_{j \in J} A_{j}\right)(x)$. Thus $\bigwedge_{j \in J} A_{j} \in \mathcal{T}_{\underline{R}}$.

(3) Take $A_{j} \in \mathcal{T}_{\underline{R}}$. Then $A_{j}=\underline{R}\left(A_{j}\right)$ for each $j \in J$. So $\left(\bigvee_{j \in J} A_{j}\right)(x)=\left(\bigvee_{j \in J} \underline{R}\left(A_{j}\right)\right)(x)=\bigvee_{j \in J} \underline{R}\left(A_{j}\right)(x)=$ $\bigvee_{j \in J}\left(\bigwedge_{y \in X}\left(A_{j}(y) \vee R^{C}(x, y)\right)\right)=\bigwedge_{y \in X}\left(\left(\bigvee_{j \in J} A_{j}\right)(y) \vee\right.$ $\left.R^{C}(x, y)\right)=\underline{R}\left(\bigvee_{j \in J} A_{j}\right)(x)$. Thus $\bigvee_{j \in J} A_{j} \in \mathcal{T}_{\underline{R}}$.

Remark 4.16. The IF topology $\mathcal{T}_{\underline{R}}$ is dual to the IF topology $\mathcal{T}_{\bar{R}}$. It follows from the fact that for $A \in \operatorname{IF}(X), A \in \mathcal{T}_{\underline{R}} \Leftrightarrow$ $A=\underline{R}(A) \Leftrightarrow A=\left(\bar{R}\left(A^{C}\right)\right)^{C} \Leftrightarrow A^{C}=\bar{R}\left(A^{C}\right) \Leftrightarrow A^{C} \in$ $\mathcal{T}_{\bar{R}}$. In addition, the IF topologies $\mathcal{T}_{\underline{R}}$ and $\mathcal{T}_{\bar{R}}$ are Alexandrov IF topologies.

Proposition 4.17. Let $(X, R)$ be an IF preordered space and $A \in \operatorname{IF}(X)$. Then

(i) $\underline{R}$ is the IF interior operator for the IF topology $\mathcal{T}_{\underline{R}}$,

(ii) $\bar{R}$ is the IF closure operator for the IF topology $\mathcal{T}_{\underline{R}}$.

Proof (i) We will show that $\operatorname{int}(A)=\underline{R}(A)$ for any $A \in$ $\operatorname{IF}(X)$. Since $\operatorname{int}(A)=\bigvee\left\{B \mid B \in \mathcal{T}_{R}, B \leq A\right\}, \operatorname{int}(A)=$ $\bigvee\{\underline{R}(B) \mid \underline{R}(B) \leq A\}$. Since $\bigvee\{\underline{R}(B) \mid \underline{R}(B) \leq A\} \leq A$, $\underline{R}(\bigvee\{\underline{R}(B) \mid \underline{R}(B) \leq A\}) \leq \underline{R}(A)$. By Theorem 2.6, $\underline{R}(\bigvee\{\underline{R}(B) \mid \underline{R}(B) \leq A\})=\bigvee\{\underline{R}(B) \mid \underline{R}(B) \leq A\}$. So $\bigvee\{\underline{R}(B) \mid \underline{R}(B) \leq A\} \leq \underline{R}(A)$. On the other hand, by $\underline{R}(A) \leq A$, we obtain $\underline{R}(A) \leq \bigvee\{\underline{R}(B) \mid \underline{R}(B) \leq A\}$. Hence $\bigvee\{\underline{R}(B) \mid \underline{R}(B) \leq A\}=\underline{R}(A)$. Therefore $\operatorname{int}(A)=$ $\underline{R}(A)$.

(ii) We will show that $\mathrm{cl}(A)=\bar{R}(A)$ for any $A \in \operatorname{IF}(X)$. Since $\operatorname{cl}(A)=\bigwedge\left\{B^{C} \mid B \in \mathcal{T}_{\underline{R}}, B^{C} \geq A\right\}, \operatorname{cl}(A)=\bigwedge\left\{\underline{R}(B)^{C}\right.$ $\left.\mid \underline{R}(B)^{C} \geq A\right\}$. By the duality, $\bar{R}(A)=\left(\underline{R}\left(A^{C}\right)\right)^{C}=$ $\left(\bigvee\left\{\underline{R}(B) \mid \underline{R}(B) \leq A^{C}\right\}\right)^{C}=\bigwedge\left\{\underline{R}(B)^{C} \mid \underline{R}(B)^{C} \geq A\right\}$. Hence $\operatorname{cl}(A)=\bar{R}(A)$.

Proposition 4.18. Let $k$ be a saturated IF closure operator on $X$. Then there exists an IF preorder $R$ on $X$ such that $k=\bar{R}$ iff

(i) $k\left(\bigvee_{j \in J} A_{j}\right)=\bigvee_{j \in J} k\left(A_{j}\right)$ for any $A_{j} \in \operatorname{IF}(X)$, and

(ii) $k((a, b) \wedge A)=(a, b) \wedge k(A)$ for any $A \in \operatorname{IF}(X)$ and $(a, b) \in I \otimes I$.
Proof Suppose that $k$ satisfies (i) and (ii). By using $k$, we define an IF relation $R$ on $X$ as

$$
R(x, y)=k\left(\chi_{\{y\}}\right)(x), \quad x, y \in X .
$$

For each $A \in \operatorname{IF}(X)$, if $x \neq y$, we have

$\left(\chi_{\{y\}} \wedge A(y)\right)(x)=\left(\chi_{\{y\}}\right)(x) \wedge A(y)=(0,1) \wedge A(y)=(0,1)$.

So $\left(\bigvee_{y \in X}\left(\chi_{\{y\}} \wedge A(y)\right)\right)(x)=\left(\chi_{\{x\}} \wedge A(x)\right)(x)=A(x)$, hence $A=\bigvee_{y \in X}\left(\chi_{\{y\}} \wedge A(y)\right)$.

For every $x \in X$, we have

$$
\begin{aligned}
\bar{R}(A)(x) & =\bigvee_{y \in X}(R(x, y) \wedge A(y)) \\
& =\bigvee_{y \in X}\left(k\left(\chi_{\{y\}}\right)(x) \wedge A(y)\right) \\
& =\bigvee_{y \in X}\left(k\left(\chi_{\{y\}}\right) \wedge A(y)\right)(x) \\
& =\bigvee_{y \in X} k\left(\left(\chi_{\{y\}}\right) \wedge A(y)\right)(x) \\
& =k\left(\bigvee_{y \in X}\left(\chi_{\{y\}}\right) \wedge A(y)\right)(x) \\
& =k(A)(x),
\end{aligned}
$$

which implies $k(A)=\bar{R}(A)$.

Conversely suppose the assumptions. Since $k$ is a Kuratowski IF closure operator, $\mathcal{T}=\left\{A^{C} \in \operatorname{IF}(X) \mid k(A)=A\right\}$ is an IF topology $\mathcal{T}$ on $X$, and it satisfies (i). By Lemma 4.9, there exists an IF preorder $R$ on $X$ with respect to the family $\mathcal{T}$. Since $k=\bar{R}, k$ satisfies (ii) by Proposition 2.4.

\section{Conflict of Interest}

No potential conflict of interest relevant to this article was reported.

\section{References}

[1] Z. Pawlak, "Rough sets," International Journal of Computer \& Information Sciences, vol. 11, no. 5, pp. 341-356, 1982. http://dx.doi.org/10.1007/BF01001956

[2] J. Kortelainen, "On relationship between modified sets, topological spaces and rough sets," Fuzzy Sets and Systems, vol. 61, no. 1, pp. 91-95, 1994. http://dx.doi.org/10. 1016/0165-0114(94)90288-7 
[3] Y. Y. Yao, "Two views of the theory of rough sets in finite universes," International Journal of Approximate Reasoning, vol. 15, no. 4, pp. 291-317, 1996. http://dx.doi. org/10.1016/S0888-613X(96)00071-0

[4] K. Qin and Z. Pei, "On the topological properties of fuzzy rough sets," Fuzzy Sets and Systems, vol. 151, no. 3, pp. 601-613, 2005. http://dx.doi.org/10.1016/j.fss.2004. 08.017

[5] D. Dubois and H. Prade, "Rough fuzzy sets and fuzzy rough sets," International Journal of General System, vol. 17, no. 2-3, pp. 191-209, 1990. http://dx.doi.org/10.1080/ 03081079008935107

[6] N. N. Morsi and M. M. Yakout, "Axiomatics for fuzzy rough sets," Fuzzy Sets and Systems, vol. 100, no. 1-3, pp. 327-342, 1998. http://dx.doi.org/10.1016/S0165-0114(97) 00104-8

[7] W. Z. Wu, J. S. Mi, and W. X. Zhang, "Generalized fuzzy rough sets," Information Sciences, vol. 151, pp. 263-282, 2003. http://dx.doi.org/10.1016/S0020-0255(02)00379-1

[8] S. P. Tiwari and A. K. Srivastava, "Fuzzy rough sets, fuzzy preorders and fuzzy topologies," Fuzzy Sets and Systems, vol. 210, pp. 63-68, 2013. http://dx.doi.org/10.1016/j.fss. 2012.06.001

[9] K. T. Atanassov, "Intuitionistic fuzzy sets," Fuzzy Sets and Systems, vol. 20, no. 1, pp. 87-96, 1986. http://dx.doi. org/10.1016/S0165-0114(86)80034-3

[10] E. Szmidt and J. Kacprzyk, "Distances between intuitionistic fuzzy sets," Fuzzy Sets and Systems, vol. 114, no. 3, pp. 505-518, 2000. http://dx.doi.org/10.1016/S01650114(98)00244-9

[11] H. Bustince and P. Burillo, "Structures on intuitionistic fuzzy relations," Fuzzy Sets and Systems, vol. 78, no. 3, pp. 293-303, 1996. http://dx.doi.org/10.1016/0165-0114(96) 84610-0

[12] L. Zhou, W. Z. Wu, and W. X. Zhang, "On intuitionistic fuzzy rough sets and their topological structures," International Journal of General Systems, vol. 38, no. 6, pp. 589616, 2009. http://dx.doi.org/10.1080/03081070802187723

[13] W. Z. Wu and L. Zhou, "On intuitionistic fuzzy topologies based on intuitionistic fuzzy reflexive and transitive relations," Soft Computing, vol. 15, no. 6, pp. 1183-1194, 2011. http://dx.doi.org/10.1007/s00500-010-0576-0

[14] D. Coker, "An introduction to intuitionistic fuzzy topological spaces," Fuzzy Sets and Systems, vol. 88, no. 1, pp. 81-89, 1997. http://dx.doi.org/10.1016/S0165-0114(96) 00076-0

[15] R. Lowen, "Fuzzy topological spaces and fuzzy compactness," Journal of Mathematical Analysis and Applications, vol. 56, no. 3, pp. 621-633, 1976. http://dx.doi.org/10. 1016/0022-247X(76)90029-9

[16] M. Mas, M. Monserrat, J. Torrens, and E. Trillas, "A survey on fuzzy implication functions," IEEE Transactions on Fuzzy Systems, vol. 15, no. 6, pp. 1107-1121, 2007. http://dx.doi.org/10.1109/TFUZZ.2007.896304

[17] B. De Baets and J. Fodor, "Residual operators of uninorms," Soft Computing, vol. 3, no. 2, pp. 89-100, 1999. http://dx.doi.org/10.1007/s005000050057

[18] P. T. Johnstone, Stone Spaces. Cambridge: Cambridge University Press, 1982.

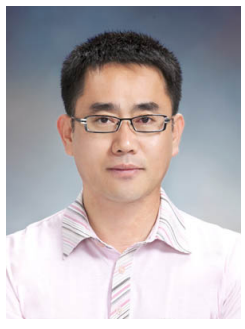

Sang Min Yun is pursuing a doctorate at Chungbuk National University. His research interests include general topology and fuzzy topology. He is a member of KIIS and KMS.

E-mail: jivesm@naver.com

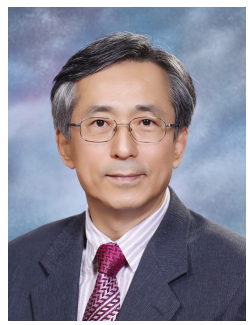

Seok Jong Lee received the M.S. and Ph.D. degrees from Yonsei University in 1986 and 1990 , respectively. He is a professor at the Department of Mathematics, Chungbuk National University since 1989 . He was a visiting scholar in Carleton University from 1995 to 1996, and Wayne State Univerisity from 2003 to 2004. His research interests include general topology and fuzzy topology. He is a member of KIIS, KMS, and CMS. He served as a general chair of the 12th International Symposium on Advanced Intelligent Systems(ISIS 2011).

E-mail: sj1@cbnu.ac.kr 